\title{
Early Myopia Onset, Predicted by First Spectacle Use, is A Risk Factor for High Myopia in Adulthood: A Retrospective Study
}

\section{Wei-Yu Chiang}

Chang Gung Memorial Hospital Kaohsiung Branch

\section{Yun-Wen Chen}

Chang Gung Memorial Hospital Kaohsiung Branch

Yu-Peng Liu

Kaohsiung Medical College: Kaohsiung Medical University

Yung-Hsun Liu

Chang Gung Memorial Hospital Kaohsiung Branch

Pei-Chang Wu ( $\nabla$ wooopc@gmail.com )

Chang Gung Memorial Hospital Kaohsiung Branch https://orcid.org/0000-0002-7201-1900

Research article

Keywords: Early age, high myopia, questionnaire, spectacle, age

Posted Date: September 22nd, 2020

DOl: https://doi.org/10.21203/rs.3.rs-73593/v1

License: (9) This work is licensed under a Creative Commons Attribution 4.0 International License. Read Full License 


\section{Abstract}

Background: To investigate the risk factors for high myopia in adulthood, especially as it relates to the age of first spectacle wearing.

Methods: A retrospective study enrolled adults aged between 20 and 45 years being invited to complete a questionnaire about age, sex, current refractive error, high myopia in parents, age at first spectacle use, refractive power of the first spectacles, and life habits at different educational stages. The association between these factors and high myopia were then evaluated and analyzed.

Results: In total, 331 participants were enrolled. Their average refractive error was -4.03 diopters, and high myopia was noted in $27.5 \%$ of the study population. Only $3.3 \%$ of participants had fathers with high myopia, while $6.0 \%$ had mothers with high myopia. The participants received their first spectacles at a mean age of 13.35 years, with a mean refractive error of -1.63 diopters.

The significant risk factors for developing high myopia in adult life were earlier age at first spectacle use $(p<0.001)$, higher refractive power of first spectacles $(p<0.001)$, mother with high myopia $(p=0.015)$, and after-school class attendance in senior high school $(p=0.018)$. Those who wore their first spectacles at $<9$ years of age were more predisposed to high myopia than those who did so at $\geqq 13$ years, with an odds ratio of 24.891, while the odds ratio for those aged between 10 and 12 years was 5.294.

Conclusions: The present study shows that earlier age at first spectacle use, higher myopic refraction of first spectacles, mothers with high myopia, and after-school class attendance in senior high school are predictive factors for high myopia in adulthood. The most important predictive factor is earlier age at first spectacle use, which emphasizes earlier myopia control is a protective factor for high myopia in adulthood.

\section{Background}

Myopia, also termed nearsightedness, is the most common ocular abnormality worldwide[1]. By 2020, the most common causes of blindness and moderate or severe vision impairment will be cataract and refractive error (reversible with spectacle correction)[2]. However, myopia is not simply a refractive error, but is also an eyesight-threatening disease [3]. Myopia, especially high myopia, is characterized by axial length elongation and can lead to several complications, such as cataract, glaucoma, myopic choroidal neovascularization, foveoschisis, macular hole, chorioretinal atrophy, peripapillary deformation, choroidal/scleral thinning, rhegmatogenous retinal detachment due to peripheral retinal tears, and even blindness [4-6]. These sight-threatening complications even affect young people of a working age, not only elderly individuals. Despite this, in the past, myopia attracted little attention from the general population or even eye care professionals. It was simply regarded as a minor inconvenience that could be managed with spectacles, contact lenses, or refractive surgical procedures[1]. However, it is now known that the condition is not simply a threat to individual vision; it is also irreversible and highly prevalent, 
confers a higher risk of the conditions mentioned above, and has been neglected by clinicians. As such, it is now considered a severe public health burden.

The prevalence of myopia demonstrates significant racial differences: the lowest rates occur in Hispanic individuals (14.2\%), followed by black (21.5\%) and white individuals (31.0\%); the highest rates occur in Chinese participants (37.2\%), according to one report[7]. The condition is particularly prevalent in East Asia, especially China, South Korea, Japan, Singapore, and Taiwan[8]. For example, in 7 to 12-year-old Taiwanese children, the annual incidence of myopia is $8 \%-18 \%$, while it is only $2.2 \%$ in 12 -year-old Australian children $[9,10]$. Nonetheless, regardless of these racial and regional differences, both myopia and high myopia are projected to increase in prevalence globally [11, 12]. In 2000, $22.9 \%$ of the world population had myopia and $2.7 \%$ had high myopia; by 2050 , the equivalent figures are estimated to be $49.8 \%$ and $9.8 \%$, respectively [11].

The pathophysiology of myopia is not yet fully determined and its etiology is multifactorial. Emmetropization is the process by which the length of the optical axis adjusts to the optical characteristics[13]. Myopization is an overshooting of emmetropization, with resultant axial elongation and thinning of the retina, choroid, and sclera, combined with further complications. The tissue that acts as the primary driver of this axial elongation has not been identified so far[13]. In clinical situation, the identified risk factors for myopia include more recent birth, higher education, more severe myopia at baseline, shorter reading distance, work that involves focusing the eye for long periods, less time outdoors, less exercise, positive history of myopia in parents, and Asian ethnicity[14-18]. Several recent studies have aimed to develop preventive strategies based on the risk factors of myopia to inform future public health efforts. Currently, myopia control can be achieved by increasing time outdoors, using atropine, and orthokeratology[19]. Because most myopia develops in childhood, particularly during the school years, children of younger age at the onset of myopia tend to have greater myopia progression[13]. Thus, slowing the onset and progression of myopia is crucial for myopia control.

Questionnaires are common method of collecting the information about a specific population; they are non-invasive and convenient to use. One study, using information from a questionnaire predicted refractive errors with reasonable sensitivity and specificity; as such, this method may assist future epidemiological studies on myopia screening [20]. In the present study, we use the recalled questionnaire to describe the prevalence of myopia in middle-aged Taiwanese individuals and further identify the risk factors for high myopia.

\section{Methods}

\section{Patients and design}

The present retrospective study used questionnaires to identify different activities and their correlation with the incidence of high myopia in the general population. Participants comprised individuals who presented for a general ocular health check-up or those who accompanied patients to the clinic at the Ophthalmology Department of Kaohsiung Chang-Gung Memorial Hospital in Taiwan, in 2014. None of 
the participants had any known ocular diseases other than refractive error, and none had not received ocular surgery or myopia control. Individuals aged between 20 and 45 years were selected based on the following criteria: stabilized refractive error, less presbyopia effects, and avoiding more recall mistakes than older-aged individuals. This study adhered to the tenets of the Declaration of Helsinki and was approved by the institutional review board of Kaohsiung Chang Gung Memorial Hospital.

The questionnaire was used to collect information about the following clinical and demographic characteristics: sex, age, current myopia degree, parents with high myopia, age at first spectacle use, refractive power of first spectacles, and life habits at different educational stages (participation in afterschool classes or sports, amount of time spent on work requiring eye focus (near work), and participation in indoor and outdoor sports in primary school, junior high school, senior high school, and university). The questionnaires were completed based on the participants' own memory and subjective information. Participants who submitted incomplete questionnaires were excluded, as were those whose first spectacles had a positive refractive power to correct hyperopia.

The right eye was selected from all participants. High myopia was defined as a refractive error of $<-6.0$ diopters.

\section{Statistical analysis}

The Kolmogorov Smirnov test was used to test for normality, as our data were not normally distributed. Continuous variables are expressed as medians (interquartile range, IQR). In the univariate analyses, comparisons between patients with and without high myopia were performed using the Mann-Whitney $U$ test for continuous variables and the Chi-square test for categorical factors. One-way repeated measure analysis of variance was used to analyze the time spent on (1) near work, (2) indoor sports, and (3) outdoor sports at different educational stages; the Greenhouse-Geisser adjustment was applied to the degrees of freedom. In the multivariate analyses, a stepwise logistic regression analysis was used to identify significant independent predictors of high myopia. Statistical significance was defined as a twotailed $p$-value $<0.05$.

\section{Results}

\section{Demographic and baseline characteristics}

In total, 331 participants completed the questionnaire and the results are listed in Table 1. Women constituted the majority of the participants $(71.3 \%)$, and the average age was 32.15 years (range: $22-$ 44 years). The average refractive error was -4.03 diopters, (range: -11.00 to 1.25 diopters), and $27.5 \%$ of the participants had high myopia. With regard to parents, $3.3 \%$ of the participants' fathers and $6.0 \%$ of their mothers had high myopia. The mean age at the prescription of first spectacle was 13 years, and the mean refractive power at that time was -1.63 diopters. In total, $71.9 \%$ of participants received their first spectacles before the age of 15 years and $35 \%$ before the age of 12 years. Of those who received their first spectacle before the age of 12 years, $58.6 \%$ developed high myopia in adulthood; in contrast, only 
$12.6 \%$ of those who received their first spectacles after the age of 12 years developed high myopia (data shown in Table 2). 
Table 1

Demographics and baseline results of all participants

\begin{tabular}{|c|c|}
\hline Clinical parameters & Results \\
\hline Total $(n)$ & 331 \\
\hline Sex (male, \%) & $95(28.7 \%)$ \\
\hline Age (years) & $32.86 \pm 4.63(22-44)$ \\
\hline Myopia (diopter) & $-4.04 \pm 2.63(-11.00 \sim 1.25)$ \\
\hline High myopia & $91(27.5 \%)$ \\
\hline Parents high myopia & $11(3.3 \%)$ \\
\hline Father & $20(6.0 \%)$ \\
\hline \multicolumn{2}{|l|}{ Mother } \\
\hline \multicolumn{2}{|l|}{ First spectacle } \\
\hline Age (years) & $13.35 \pm 3.80(2 \sim 30)$ \\
\hline Before 15 years old & $238(71.9 \%)$ \\
\hline Before 12 years old & $116(35.0 \%)$ \\
\hline Diopter & $-1.63 \pm 0.99(-5 \sim-0.5)$ \\
\hline \multicolumn{2}{|l|}{ Primary school } \\
\hline Near work (hr/wk) & $19.04 \pm 15.68(0 \sim 106)$ \\
\hline Outdoor sport (hr/wk) & $4.13 \pm 7.31(0 \sim 50)$ \\
\hline Indoor sport (hr/wk) & $1.31 \pm 3.49(0 \sim 25)$ \\
\hline After-school classes attendance & $130(39.3)$ \\
\hline Sport team & $46(13.9)$ \\
\hline \multicolumn{2}{|l|}{ Junior high school } \\
\hline Near work (hr/wk) & $22.22 \pm 16.55(0 \sim 105)$ \\
\hline Outdoor sport (hr/wk) & $2.71 \pm 6.21(0 \sim 72)$ \\
\hline Indoor sport (hr/wk) & $0.97 \pm 3.28(0 \sim 48)$ \\
\hline After-school classes attendance & $250(75.5 \%)$ \\
\hline Sport team & $19(5.7 \%)$ \\
\hline \multicolumn{2}{|l|}{ Senior high school } \\
\hline Near work (hr/wk) & $23.60 \pm 18.38(0 \sim 105)$ \\
\hline
\end{tabular}




\begin{tabular}{|ll|}
\hline Clinical parameters & Results \\
\hline Outdoor sport $(\mathrm{hr} / \mathrm{wk})$ & $1.97 \pm 4.34(0 \sim 50)$ \\
\hline Indoor sport $(\mathrm{hr} / \mathrm{wk})$ & $0.74 \pm 2.18(0 \sim 22)$ \\
\hline After-school classes attendance & $150(45.3)$ \\
\hline Sport team & $13(3.9 \%)$ \\
\hline University & \\
\hline Near work $(\mathrm{hr} / \mathrm{wk})$ & $26.60 \pm 19.34(0 \sim 132)$ \\
\hline Outdoor sport $(\mathrm{hr} / \mathrm{wk})$ & $1.87 \pm 3.96(0 \sim 35)$ \\
\hline Indoor sport (hr/wk) & $1.34 \pm 6.74(0 \sim 105)$ \\
\hline After-school classes attendance & $20(6.0 \%)$ \\
\hline Sport team & $14(4.2 \%)$ \\
\hline
\end{tabular}

Table 2

The first spectacle age and the high myopia prevalence in adulthood

\begin{tabular}{|ll|}
\hline The first spectacle age & High myopia in adulthood \\
\hline If before 12 years & $58.6 \%$ \\
\hline If after 12 years & $12.6 \%$ \\
\hline If before 15 years & $35.7 \%$ \\
\hline If after 15 years & $8.5 \%$ \\
\hline
\end{tabular}

Figure shows data regarding life habits at the four different educational stages, that is, the time spent on (1) near work, (2) outdoor sport, and (3) indoor sport. The time spent on near work showed significant linear increase from from primary school to university $(p<0.001)$. In contrast, the time spent on outdoor sports showed a significant linear decrease $(p<0.001)$, while the time spent on indoor sport showed a significant quadratic trend $(p=0.013)$ with lowest time in senior high school.

\section{The comparison between high myopia and non-high myopia}

Next, we compared participants with high myopia to those who had non-high myopia, as shown in Table 3. Age and sex were not significantly different. Although the proportion of fathers with high myopia were not significantly different, mothers with high myopia were significantly more in the high myopia group ( 12.1 vs. $3.8 \%, p=0.004)$. The age at first spectacle use was significantly younger in the high myopia group ( 11.05 vs. $14.36 y$ ears; $p<0.001)$, and high myopia group showed a higher percentage of participants who used spectacles before the age of 12 years $(74.7$ vs. $23.1 \%, p<0.001)$. The refractive power of the participants' first spectacle was also higher in the high myopia group (-2.28 vs. -1.38 
diopters; $p$ < 0.001). In the primary school period, the high myopia group spent more time on near work (21.21 vs. 18.22 hours/week, respectively; $p=0.042)$; they also showed less indoor sports time (0.54 vs. 1.60 hours/week; $p=0.034$ ) and less outdoor sports time ( 2.92 vs. 4.59 hours/week; $p=0.033)$ during the same period. In junior high school, the high myopia group tended to spend more time on near work (24.23 vs. 21.45 hours/week; $p=0.053)$. The high myopia group spent significantly more time on near work in both senior high school ( 26.45 vs. 22.52 hours/week; $p=0.029)$ and university ( 29.73 vs. 25.42 hours/week; $p=0.007$ ). After-school class attendance in senior high school was significantly higher in the high myopia group (54.9 vs. $41.7 \%$; $=0.030$ ). 
Table 3

Comparison between high myopia and non-high myopia

\begin{tabular}{|c|c|c|c|}
\hline Clinical parameters & $\begin{array}{l}\text { High myopia } \\
(n=91)\end{array}$ & $\begin{array}{l}\text { Non-high myopia } \\
(n=240)\end{array}$ & $p$ value \\
\hline Age (years) & $32.51 \pm 4.21$ & $33.00 \pm 4.78$ & 0.334 \\
\hline Sex (male) & $29(31.9 \%)$ & $66(27.5 \%)$ & 0.433 \\
\hline Current myopia (diopter) & $-7.42 \pm 1.61$ & $-2.85 \pm 1.64$ & $<0.001^{*}$ \\
\hline \multicolumn{4}{|l|}{ Parents high myopia } \\
\hline Father & $4(4.4 \%)$ & $7(2.9 \%)$ & 0.503 \\
\hline Mother & $11(12.1 \%)$ & $9(3.8 \%)$ & $0.004^{\star}$ \\
\hline \multicolumn{4}{|l|}{ First glasses } \\
\hline Age (years) & $11.05 \pm 2.60$ & $14.36 \pm 3.76$ & $<0.001^{*}$ \\
\hline Age before 12 years old & $68(74.7 \%)$ & $48(23.1 \%)$ & $<0.001^{*}$ \\
\hline Age before 15 years old & $85(94.4 \%)$ & $153(73.9 \%)$ & $<0.001 *$ \\
\hline Diopter & $-2.28 \pm 0.89$ & $-1.38 \pm 0.91$ & $<0.001^{*}$ \\
\hline \multicolumn{4}{|l|}{ Primary school } \\
\hline Near work (hr/wk) & $21.21 \pm 16.16$ & $18.22 \pm 15.45$ & $0.042^{*}$ \\
\hline Indoor sports (hr/wk) & $0.54 \pm 1.22$ & $1.60 \pm 3.99$ & $0.034^{\star}$ \\
\hline Outdoor sports (hr/wk) & $2.92 \pm 5.73$ & $4.59 \pm 7.78$ & $0.033^{*}$ \\
\hline After-school classes attendance & $37(40.7 \%)$ & $93(38.8 \%)$ & 0.751 \\
\hline Sport team & $11(12.1 \%)$ & $35(14.6 \%)$ & 0.558 \\
\hline \multicolumn{4}{|l|}{ Junior high school } \\
\hline Near work (hr/wk) & $24.23 \pm 15.82$ & $21.45 \pm 16.79$ & 0.053 \\
\hline Indoor sports (hr/wk) & $0.49 \pm 1.08$ & $1.15 \pm 3.79$ & 0.188 \\
\hline Outdoor sports (hr/wk) & $2.45 \pm 5.11$ & $2.81 \pm 6.59$ & 0.314 \\
\hline After-school classes attendance & $74(81.3 \%)$ & $176(73.3 \%)$ & 0.131 \\
\hline Sport team & $6(6.6 \%)$ & $13(5.4 \%)$ & 0.681 \\
\hline \multicolumn{4}{|l|}{ Senior high school } \\
\hline Near work (hr/wk) & $26.45 \pm 18.01$ & $22.52 \pm 18.44$ & $0.029 *$ \\
\hline Indoor sports (hr/wk) & $0.57 \pm 1.47$ & $0.81 \pm 2.39$ & 0.583 \\
\hline
\end{tabular}




\begin{tabular}{|llll|}
\hline Clinical parameters & $\begin{array}{l}\text { High myopia } \\
(\mathbf{n = 9 1 )}\end{array}$ & $\begin{array}{l}\text { Non-high myopia } \\
(\mathbf{n}=\mathbf{2 4 0})\end{array}$ & p value \\
\hline Outdoor sports (hr/wk) & $1.89 \pm 3.26$ & $2.00 \pm 4.69$ & 0.767 \\
\hline After-school classes attendance & $50(54.9 \%)$ & $100(41.7 \%)$ & $0.030^{*}$ \\
\hline Sport team & $4(4.4 \%)$ & $9(3.8 \%)$ & 0.787 \\
\hline University & & & \\
\hline Near work (hr/wk) & $29.73 \pm 17.07$ & $25.42 \pm 20.04$ & $0.007^{\star}$ \\
\hline Indoor sports (hr/wk) & $0.64 \pm 1.40$ & $1.61 \pm 7.85$ & 0.766 \\
\hline Outdoor sports (hr/wk) & $1.85 \pm 4.27$ & $1.88 \pm 3.84$ & 0.901 \\
\hline After-school classes attendance & $6(6.6 \%)$ & $14(5.8 \%)$ & 0.796 \\
\hline Sport team & $3(3.3 \%)$ & $11(4.6 \%)$ & 0.604 \\
\hline
\end{tabular}

\section{Multivariate analysis for risk factors of high myopia}

To identify the risk factors of high myopia, several significant factors in Table 3 were analyzed by logistic regression (Table 4). After multivariate analysis, the identified risk factors for high myopia were early age at first spectacle use $(p<0.001)$, high refractive power of first spectacles $(p<0.001)$, high myopia in mother $(p=0.015)$, and after-school class attendance in senior high school $(p=0.018)$. When all participants were divided into men and women, the risk factors for high myopia exhibited by men were earlier age of first spectacle use (odds ratio [OR]: 0.669, 95\% confidence interval [Cl] 0.532-0.840; $p=$ 0.001 ) and indoor sport time in university (OR: $0.660,95 \% \mathrm{Cl}: 0.434-1.004 ; \mathrm{p}=0.052$ ). In contrast, women showed the following risk factors: early age at first spectacle use (OR: 0.604, 95\% Cl: 0.492-0.743; $p<$ 0.001 ), high refractive power of first spectacles (OR: $0.981,95 \% \mathrm{Cl}: 0.975-0.988 ; p<0.001$ ), high myopia in mother (OR: $11.391,95 \% \mathrm{Cl}: 1.732-74.903 ; p=0.011)$, and indoor sport time in university (OR: 1.408, $95 \% \mathrm{Cl}: 1.021-1.942 ; \mathrm{p}=0.037$ ) (data not shown in table).

Table 4

Logistic regression analysis for high myopia

\begin{tabular}{|l|l|l|l|}
\hline & Odds ratio $95 \%$ Cl & $p$ value \\
\hline 1 st spectacle age & 0.649 & $0.564-0.747$ & $<0.001$ \\
\hline 1st spectacle refractive power & 0.986 & $0.982-0.991$ & $<0.001$ \\
\hline Mother high myopia (yes vs. no) & 5.591 & $1.401-22.3160 .015$ \\
\hline After-school classes attendance in senior high school 2.488 & $1.158-4.620$ & 0.018 \\
\hline Cl confidence interval & & & \\
\hline
\end{tabular}

The effect of the first spectacle age on high myopia in adulthood

The age at first spectacle use had a significant impact on high myopia prediction; thus, we analyzed the OR for high myopia at different ages at first spectacle use (Table 5). Participants who were younger than 9 years and between 10 and 12 years at their first spectacle use had an OR of 24.891 and 5.294 
compared with those who were aged older than 13 years, respectively. This result showed that younger age at first spectacle use was a predictor of more severe myopia progression in the future.

\section{Table 5}

Logistic regression analysis of the age of the first spectacle

for high myopia

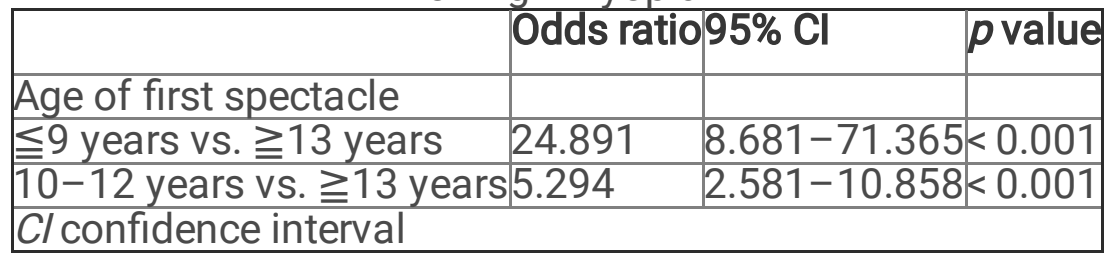

\section{Discussion}

The prevalence of myopia is increasing worldwide and is particularly high in East Asian countries. It can lead to axial elongation and induce various vision-threatening complications, as mentioned in the introduction, even in individuals of working age. Therefore, myopia should not be ignored, and prevention is better than treatment. Both hereditary and environmental factors contribute to myopia progression; these were analyzed in the present study and are interpreted in the following paragraphs.

With regard to parents' myopia status, the rate of high myopia in the participants (27.5\%) was much higher compared with fathers with high myopia (3.3\%) and mothers with high myopia (6.0\%), which is compatible with previous reports that the prevalence of both myopia and high myopia is increasing rapidly[12]. Family history of myopia is a risk factor for adult-onset myopia[21-23], and Parssinen et al indicated that women with myopic parents showed higher refractive power and faster/earlier myopia progression than did those with non-myopic parents [18]. Our results indicated that mothers may contribute more on hereditary myopia compared with fathers. After subgroup analysis based on sex, high myopia in mothers was only a risk factor for high myopia in women but not in men. Thus, further studies are necessary to identify the roles played by sex in hereditary myopia. Conversely, the great difference in myopia prevalence between the two generations suggests that genetics does not have a major effect on myopia prevalence; environmental factors may be the major cause.

One recent meta-analysis showed that more time spent on near work was associated with higher odds of myopia[17]. Higher educational level is associated with more myopic refraction[14, 24], which may be linked to longer time spent on near work. This may partially explain why there is high myopia prevalence in East Asia, where students spend much more time on studying and after-school classes because there is fierce competition for higher education. In the present study, univariate analyses showed that the high myopia group spent more time on near work at all four educational stages, and multivariate analysis indicated that high myopia group undertook more after-school class in senior high school.

In contrast, longer time spent outdoors is a protective factor for myopia[10,25,26]. This is an important factor in public health efforts. Further studies have found that the bright light outdoors increase dopamine receptor activity, with resultant decreases in myopic shift and ocular elongation[27-29]. In Taiwan, after outdoor activities were implemented in the Taiwan Student Vision Care Program, the rate of vision impairment decreased[30]. Recently, Wu et al found that activities undertaken in lower outdoor light 
intensity, such as in hallways or under trees, also provide some protective effects on myopia development[31]. In the present study, although this did not reach significance in multivariate analyses, both outdoor and indoor sports time were significantly less in primary school in high myopia group (Table 3).

The age at first spectacle use has been discussed in previous studies. In a UK-based study, almost half of the individuals with myopia wore glasses only after the age of 17 years [32]. Rafael et al reported that the subjects who were older at their first spectacle use tended to develop lower refractive error, but this relationship was not significant[33]. Another study indicated that subjects who developed myopia after the age of 20 years had low myopia[34]. Wearing spectacles to correct myopic refractive error is more common in East Asia than in Western countries. The present study revealed that the mean refractive error of the first prescription in Taiwan was - 1.62 diopters, at a mean age of 13 years. This is compatible with the clinical guidelines for childhood vision care in Taiwan, which suggests that the first spectacles should be prescribed once children having myopia of around -1.50 diopter who need refractive assistance in life. However, one study showed that refractive cutoffs $\leq-0.75$ diopters of myopia showed significantly greater improvement in vision than children failing to meet the criteria[35]. Table 4 shows that younger age at first spectacle use was a predictor of more severe myopia progression in the future, with a high OR. Based on the results, another interesting issue is that should we postpone glasses use or under-correction for myopic children who need refractive assistance. First, it's an important fact that early age at first spectacle use is the result of early myopia onset. In this study, we use the age of wearing first spectacle to estimate the age of myopia onset. Early age at first spectacle use indicated the early age of myopia onset. Secondly, as for the consideration of glasses use to control myopia progression, this question is still controversial and under investigation. The manipulations of optic correction in spectacles, including undercorrection, full correction, multifocal or bifocal, are investigated to control myopia progression, but several studies show a variety of results, ranging from decreasing to worsening myopia progression [3640].

Based on the present results, high myopia is much more prevalent now than in the previous generation and mother may play a more important role, which has not been fully described in available literature. Compared with non-high myopia, the high myopia group had early age and higher refractive power of the first spectacle used as predisposing factors, less outdoor activity in primary school and longer near-work time in all educational stages, primary through tertiary. After multivariate analyses, early age and higher refractive power of the first spectacle, mother with high myopia, and after-school classes in senior high school were significant risk factors for high myopia.

At this point, we would like to emphasize the feasibility of questionnaire use in epidemiological studies and the importance of early myopia control. The present study was the first report to analyze the risk associated with first spectacle use at different ages regarding final myopic refractive error. Early age at first spectacle use is linked to earlier myopia onset. These results demonstrate that earlier age at myopia onset increases the risk of developing high myopia in adulthood. That is, for myopia onset, latter would be better. 
There are some limitations in the current study. First, the questionnaire collected self-reported data, so the memory recall of participants was a major limitation. Second, female participants (71.3\%) were more than male participants $(28.7 \%)$. In this regard, the participants were selected from among those who came for a general ocular health check-up or accompanied patients. There are 3 reasons to explain sex disparity. One is that females are more careful about their health; another is that females are more willing to accompany their family or friends to the hospital; the third reason is that female were more willing to receive our invitation and complete the questionnaire. Thirdly, the participants' purposes of spectacle use may be not all for myopia, perhaps for astigmatism. Lastly, the time of after-school classes and sport team attendance did not be quantified. The result indicated that after school class attendance only in senior high school was associated with high myopia but not other periods. Perhaps the time and strength of after-school classes in senior high school were much more and higher than other periods, because the stress of university entry system was upmost in senior high school. Further prospective longitudinal studies with larger samples and objective refraction examination are warranted to precisely identify the risk factors for myopia onset and progression.

\section{Conclusions}

The present study indicates the predictive factors for high myopia, including earlier age at first spectacle use, higher initial refractive power of the spectacles, high myopia in mother, and after-school class attendance in senior high school. In brief, an earlier age at first spectacle use should be considered as a warning sign for the development of high myopia in later life, and emphasis should be placed on early myopia control.

\section{Abbreviations}

OR: odds ratio; SD: standard deviation;

\section{Declarations}

\section{Acknowledgements}

The authors appreciated the Biostatistics Center, Kaohsiung Chang Gung Memorial Hospital for statistics work.

\section{Funding}

None of the following authors have any proprietary interests or conflicts of interest related to this submission: Wei-Yu Chiang, Yun-Wen Chen, Yu-Peng Liu, Yung-Hsun Liu, Pei-Chang Wu

\section{Availability of data and materials}

The datasets used and/or analyzed during the current study are available 
from the corresponding author on reasonable request.

\section{Authors' contributions}

All authors have participated directly in planning and execution of the work and have approved the final version of the manuscript. WYC: acquisition and analysis of data, drafting and writing the article; YWC: acquisition of data, drafting and writing the article; YPL: helped to draft the manuscript; YHL: acquisition and analysis of data; PCW: participated in the design of the study, analysis of data.

\section{Ethics approval and consent to participate}

This study adhered to the tenets of the Declaration of Helsinki, and was approved by the Institutional Review Board of Chang Gung Memorial Hospital (study reference number: 105-0011D). The Institutional Review Board granted a waiver of consent for this study.

\section{Consent for publication}

Not applicable.

\section{Competing interests}

The authors declare that they have no competing interests.

\section{References}

1. Chua J, Wong TY. Myopia-The Silent Epidemic That Should Not Be Ignored. JAMA Ophthalmol. 2016; 134:1363-1364.

2. Flaxman SR, Bourne RRA, Resnikoff S, Ackland P, Braithwaite T, Cicinelli MV, Das A, Jonas JB, Keeffe $\mathrm{J}$, Kempen JH et al. Global causes of blindness and distance vision impairment 1990-2020: a systematic review and meta-analysis. Lancet Glob Health. 2017; 5:e1221-e1234.

3. Wu PC, Huang HM, Yu HJ, Fang PC, Chen CT. Epidemiology of Myopia. Asia Pac J Ophthalmol (Phila). 2016; 5:386-393.

4. Cho BJ, Shin JY, Yu HG. Complications of Pathologic Myopia. Eye Contact Lens. 2016; 42:9-15.

5. Ikuno Y. OVERVIEW OF THE COMPLICATIONS OF HIGH MYOPIA. Retina. 2017; 37:2347-2351.

6. Flitcroft DI. The complex interactions of retinal, optical and environmental factors in myopia aetiology. Prog Retin Eye Res. 2012; 31:622-660.

7. Pan CW, Klein BE, Cotch MF, Shrager S, Klein R, Folsom A, Kronmal R, Shea SJ, Burke GL, Saw SM, Wong TY et al. Racial variations in the prevalence of refractive errors in the United States: the multiethnic study of atherosclerosis. Am J Ophthalmol. 2013; 155:1129-1138.

8. Ding BY, Shih YF, Lin LLK, Hsiao CK, Wang IJ. Myopia among schoolchildren in East Asia and Singapore. Surv Ophthalmol. 2017; 62:677-697. 
9. French AN, Morgan IG, Burlutsky G, Mitchell P, Rose KA. Prevalence and 5- to 6-year incidence and progression of myopia and hyperopia in Australian schoolchildren. Ophthalmology. 2013; 120:14821491.

10. Wu PC, Tsai CL, Wu HL, Yang YH, Kuo HK. Outdoor activity during class recess reduces myopia onset and progression in school children. Ophthalmology. 2013; 120:1080-1085.

11. Holden BA, Fricke TR, Wilson DA, Jong M, Naidoo KS, Sankaridurg P, Wong TY, Naduvilath TJ, Resnikoff S. Global Prevalence of Myopia and High Myopia and Temporal Trends from 2000 through 2050. Ophthalmology. 2016; 123:1036-1042.

12. Dolgin E. The myopia boom. Nature. 2015; 519:276-278.

13. Wu PC, Chuang MN, Choi J, Chen H, Wu G, Ohno-Matsui K, Jonas JB, Cheung CMG. Update in myopia and treatment strategy of atropine use in myopia control. Eye (Lond). 2019; 33:3-13.

14. Williams KM, Bertelsen G, Cumberland P, Wolfram C, Verhoeven VJ, Anastasopoulos E, Buitendijk GH, Cougnard-Gregoire A, Creuzot-Garcher C, Erke MG et al. Increasing Prevalence of Myopia in Europe and the Impact of Education. Ophthalmology. 2015; 122:1489-1497.

15. Hsu CC, Huang N, Lin PY, Fang SY, Tsai DC, Chen SY, Tsai CY, Woung LC, Chiou SH, Liu CJ. Risk factors for myopia progression in second-grade primary school children in Taipei: a populationbased cohort study. Br J Ophthalmol. 2017; 101:1611-1617.

16. Theophanous C, Modjtahedi BS, Batech M, Marlin DS, Luong TQ, Fong DS. Myopia prevalence and risk factors in children. Clin Ophthalmol. 2018; 29:1581-1587.

17. Huang HM, Chang DS, Wu PC. The Association between Near Work Activities and Myopia in ChildrenA Systematic Review and Meta-Analysis. PLoS One. 2015; 10:e0140419.

18. Parssinen 0 , Kauppinen $M$. What is the influence of parents' myopia on their children's myopic progression? A 22-year follow-up study. Acta Ophthalmol. 2016; 94:579-585.

19. Leo SW. Current approaches to myopia control. Curr Opin Ophthalmol. 2017; 28:267-275.

20. Ip J, Robaei D, Rochtchina E, Rose K, Smith W, Wang JJ, Mitchell P. Can information on the purpose of spectacle use and age at first use predict refractive error type? Ophthalmic Epidemiol. 2007; 14:8892.

21. Iribarren R IG, Castagnola MM, Balsa A, Cerrella MR, Armesto A, Fornaciari A, Pförtner T.. Family history and reading habits in adult-onset myopia. Curr Eye Res. 2002; 25:309-315.

22. Liang CL, Yen E, Su JY, Liu C, Chang TY, Park N, Wu MJ, Lee S, Flynn JT, Juo SH. Impact of family history of high myopia on level and onset of myopia. Invest Ophthalmol Vis Sci. 2004; 45:3446-3452.

23. Kurtz D, Hyman L, Gwiazda JE, Manny R, Dong LM, Wang Y, Scheiman M. Role of parental myopia in the progression of myopia and its interaction with treatment in COMET children. Invest Ophthalmol Vis Sci. 2007; 48:562-570.

24. Mirshahi A, Ponto KA, Hoehn R, Zwiener I, Zeller T, Lackner K, Beutel ME, Pfeiffer N. Myopia and level of education: results from the Gutenberg Health Study. Ophthalmology. 2014; 121:2047-2052. 
25. French AN, Ashby RS, Morgan IG, Rose KA. Time outdoors and the prevention of myopia. Exp Eye Res. 2013; 114:58-68.

26. He M, Xiang F, Zeng Y, Mai J, Chen Q, Zhang J, Smith W, Rose K, Morgan IG. Effect of Time Spent Outdoors at School on the Development of Myopia Among Children in China: A Randomized Clinical Trial. JAMA. 2015; 314:1142-1148.

27. Morgan IG, Ashby RS. Bright Light Blocks the Development of Form Deprivation Myopia in Mice, Acting on D1 Dopamine Receptors. Invest Ophthalmol Vis Sci. 2017; 58:2317.

28. Chen S, Zhi Z, Ruan Q, Liu Q, Li F, Wan F, Reinach PS, Chen J, Qu J, Zhou X. Bright Light Suppresses Form-Deprivation Myopia Development With Activation of Dopamine D1 Receptor Signaling in the ON Pathway in Retina. Invest Ophthalmol Vis Sci. 2017; 58:2306-2316.

29. Wang Y, Ding H, Stell WK, Liu L, Li S, Liu H, Zhong X. Exposure to sunlight reduces the risk of myopia in rhesus monkeys. PLoS One. 2015; 10:e0127863.

30. Wu PC, Chang LC, Niu YZ, Chen ML, Liao LL, Chen CT. Myopia prevention in Taiwan. Annals of Eye Science. 2018, 3(12).

31. Wu PC, Chen CT, Lin KK, Sun CC, Kuo CN, Huang HM, Poon YC, Yang ML, Chen CY, Huang JC et al. Myopia Prevention and Outdoor Light Intensity in a School-Based Cluster Randomized Trial. Ophthalmology. 2018; 125:1239-1250.

32. Williams KM, Hysi PG, Nag A, Yonova-Doing E, Venturini C, Hammond CJ. Age of myopia onset in a British population-based twin cohort. Ophthalmic Physiol Opt. 2013; 33:339-345.

33. Iribarren R, Cortinez MF, Chiappe JP, Chiappe JP. Age of first distance spectacle prescription for manifest hyperopia. Curr Eye Res. 2010; 35:385-388.

34. Iribarren R, Cortinez MF, Chiappe JP. Age of first distance prescription and final myopic refractive error. Ophthalmic Epidemiol. 2009; 16:84-89.

35. Congdon NG, Patel N, Esteso P, Chikwembani F, Webber F, Msithini RB, Ratcliffe A. The association between refractive cutoffs for spectacle provision and visual improvement among school-aged children in South Africa. Br J Ophthalmol. 2008; 92:13-18.

36. Sun YY, Li SM, Li SY, Kang MT, Liu LR, Meng B, Zhang FJ, Millodot M, Wang N. Effect of uncorrection versus full correction on myopia progression in 12-year-old children. Graefes Arch Clin Exp Ophthalmol. 2017; 255:189-195.

37. Li SM, Li SY, Liu LR, Guo JY, Chen W, Wang NL, Millodot M. Full correction and Undercorrection of Myopia Evaluation Trial: design and baseline data of a randomized, controlled, double-blind trial. Clin Exp Ophthalmol. 2013; 41:329-338.

38. Chung K, Mohidin N, O'Leary DJ. Undercorrection of myopia enhances rather than inhibits myopia progression. Vision Res. 2002; 42:2555-2559.

39. Li SY, Li SM, Zhou YH, Liu LR, Li H, Kang MT, Zhan SY, Wang N, Millodot M. Effect of undercorrection on myopia progression in 12-year-old children. Graefes Arch Clin Exp Ophthalmol. 2015; 253:13631368. 
40. Adler D, Millodot M. The possible effect of undercorrection on myopic progression in children. Clin Exp Optom. 2006; 89:315-321.

\section{Figures}

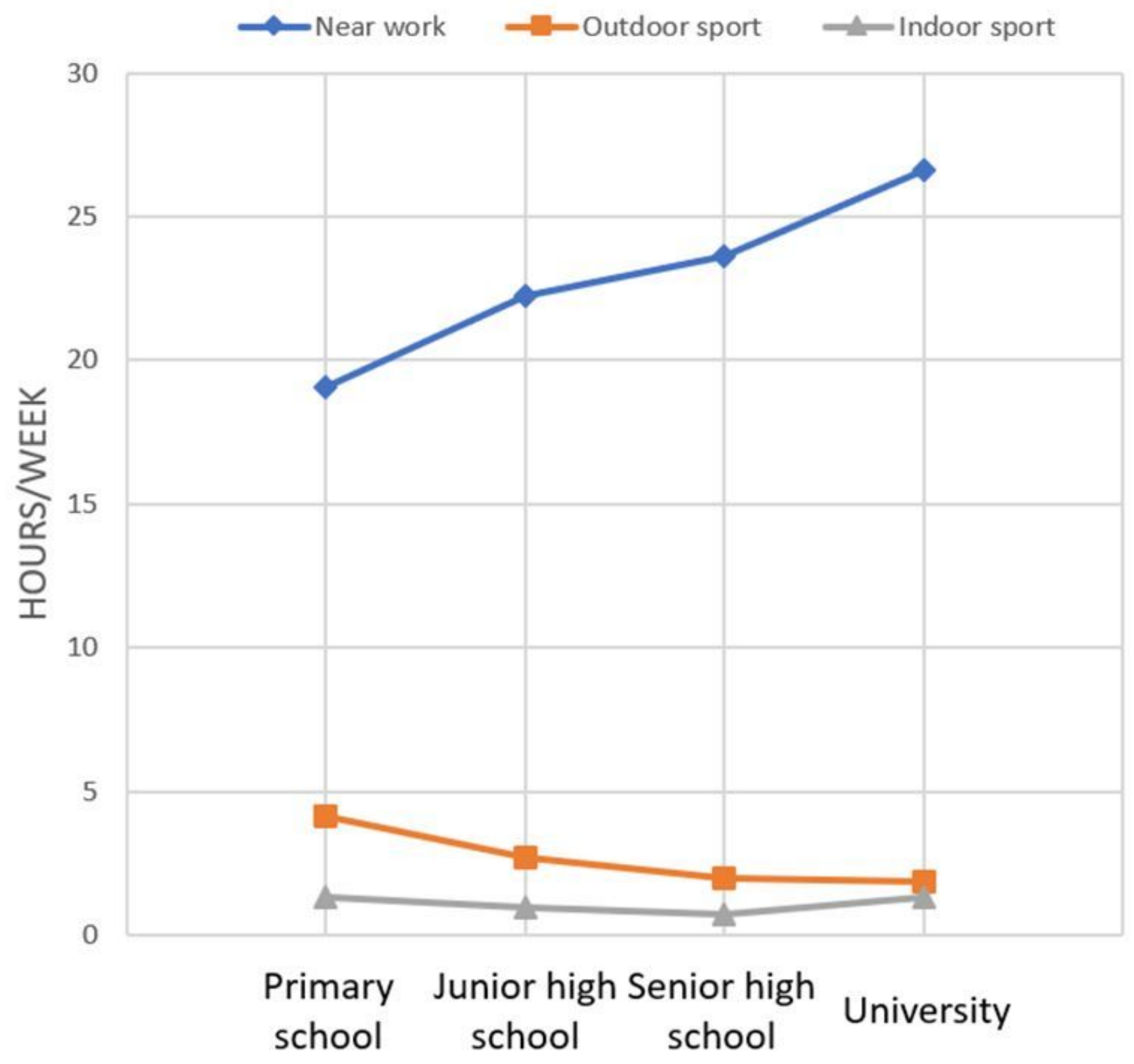

Figure 1

The mean of the time utilized engaging in near work, outdoor sport, and indoor sport in four different educational stages. The time utilized engaging in work that requires close eye concentration. of near work shows a significant positive linear trend $(p<0.001)$, outdoor sport shows a significant negative linear 
trend $(p<0.001)$. The time of indoor sport showed a significant quadratic trend $(p=0.013)$ with the lowest time in senior high school. Error bars indicate 1 standard deviation (SD).

\section{Supplementary Files}

This is a list of supplementary files associated with this preprint. Click to download.

- Questionnaire.docx 